\title{
Draft genome of Elstera litoralis, a freshwater epilithic biofilm associated bacterium from littoral zone of Lake Constance
}

\author{
Monali C. Rahalkar a,*, Soham Pore ${ }^{\mathrm{a}, 1}$, Preeti Arora ${ }^{\mathrm{a}, 1}$, Pranitha Pandit ${ }^{\mathrm{a}, 1}$, Neelam Kapse ${ }^{\mathrm{a}, 1}$, Rahul Bahulikar ${ }^{\mathrm{b}, 2}$, \\ Bernhard Schink ${ }^{\mathrm{b}}$, Prashant K. Dhakephalkar ${ }^{\mathrm{a}, 1}$ \\ a Bioenergy Group, MACS Agharkar Research Institute, G.G. Agarkar Road, Pune 411004, Maharashtra, India \\ ${ }^{\mathrm{b}}$ Faculty of Biology, University of Konstanz, D-78457 Konstanz, Germany
}

Keywords:

Elstera litoralis

Epilithic biofilms

Lake Constance

Draft genome

\begin{abstract}
A B S T R A C T
Elstera litoralis, is a Rhodospirillaceae member which was isolated from the littoral zone of Lake Constance from a stone biofilm using diatom extracellular polymeric substances (EPS) as C source. We present here the draft ge nome of $E$. litoralis which has a genome size of $3.83 \mathrm{Mb}$ and $61.2 \% \mathrm{G}+\mathrm{C}$ content. Genome analysis indicated uti lization of multiple $C$ substrates explaining its heterotrophic lifestyle as a bacterium present in natural biofilms. Further comparative genome analysis of Elstera with other members of Rhodospirillaceae would be helpful to un derstand the evolutionary relationships and divergence of hydrobacteria from terrabacteria.
\end{abstract}

Elstera is a recently described genus within the family Rhodospirillaceae, of alpha proteobacteria with a single species, Elstera litoralis (Rahalkar et al., 2012). When isolated, it was most related to Azospirillum spp. ( 90 92\%) based on its 16S rRNA gene sequence, however after the description of a lake bacterium, Lacibacterium aquatile (Sheu et al., 2013), E. litoralis was found to be most related to L. aquatile on the basis of $16 \mathrm{~S}$ rRNA gene by $94 \%$ similarity. E. litoralis was isolated from epilithic biofilms from the littoral zone of Lake Con stance (Rahalkar et al., 2012). Lake Constance is the second largest freshwater lake in Europe and is characterized by a littoral zone com prising of stones covered with biofilms. In our previous studies, we have shown that diatom EPS (Bahulikar and Kroth, 2008) can act as C source for heterotrophic bacteria (Bruckner et al., 2008). E. litoralis was isolated as one of the most dominant heterotroph from such a bio film using diatom EPS as the $C$ source in a following study (Rahalkar et al., 2012). Diatoms and bacteria are the dominant members of the biofilm community in the littoral zone of Lake Constance where EPS produced by diatoms can be used by heterotrophic organisms as a car bon source (Bruckner et al., 2008). To the best of our knowledge, there are no reported complete or draft genome sequences of organisms iso lated from freshwater epilithic biofilms. Here we announce the draft

\footnotetext{
* Corresponding author. Tel.: +9120 25653680; fax: +91202651542.

E-mail addresses: monalirahalkar@aripune.org, monarahul2002@yahoo.com (M.C. Rahalkar).

${ }^{1}$ Tel.: + 9120 25653680; fax: + 91202651542.

2 Present address: BAIF Development Research Foundation, Central Research Station, Uruli Kanchan, Pune, India.
}

genome of $E$. litoralis Dia $1^{\mathrm{T}}$. The whole genome helped us in under standing the evolution and life strategies of this organism which is present naturally in complex environment such as a stone biofilm, occurring between land and water.

The genome of $E$. litoralis was sequenced using the Ion Torrent PGM (200 bp) with the $316^{\mathrm{TM}}$ sequencing chip using the manufacturer's in structions (Life Technologies, USA). De novo assembly was performed using the MIRA 4.0.5 assembler (Chevreux et al., 1999) which generated 1012 contigs, with N50 of 8551 bp and N90 of 2026 bp. This data was then submitted to the Rapid Annotation using Subsystem Technology (RAST server) (Aziz et al., 2008; Overbeek et al., 2014) and NCBI genome sequence database. RAST server was used for subsystem analysis where the subsystem coverage in this case was $46 \%$ belonging to 408 subsys tems. Closest genome match of the genome was with Rhodospirillum centenum (genome ID: 414684.4) with 539 score, an aquatic bacterium in the same family. It was revealed that genome size of $E$. litoralis was $383,027 \mathrm{Mb}$ with a $\mathrm{G}+\mathrm{C}$ content of $61.2 \%$. Annotation by NCBI prokary otic genome annotation pipeline (PGAAP) http://www.ncbi.nlm.nih. gov/genome/annotation_prok/revealed the presence of 1012 contigs with 3310 coding DNA sequences, 57 tRNAs and 14 rRNA (5S, 16S and 23S) with a single rRNA operon (Table 1).

The absence of any photosynthetic apparatus and genes of Calvin cycle was consistent with that of Azospirillum species in contrary to most of the other Rhodospirillaceae members (Wisniewski Dye et al., 2011; Baldani et al., 2014). As E. litoralis dwells in stone biofilms, prima ry production may not be necessary function, as these biofilms are mainly dominated by diatoms and algae that may provide organic car bon for heterotrophic community members. Thus, E. litoralis may be 
Table 1

Genome features of Elstera litoralis.

\begin{tabular}{ll}
\hline Attributes & Values \\
\hline Genome size & $3.83 \mathrm{Mb}$ \\
Contigs & 1012 \\
$\mathrm{G}+\mathrm{C}$ content & $61.2 \%$ \\
CDs & 3310 \\
tRNA & 57 \\
rRNA & $14(5 \mathrm{~S}, 16 \mathrm{~S}, 23 \mathrm{~S})$ \\
ncRNA & 1 \\
\hline
\end{tabular}

adapted to a heterotrophic metabolism, in contrast to the autotrophic lifestyle observed in other members of the family. This was reflected in the detection of genes involved in the utilization of variety of sub strates, including sugars, amino sugars, sugar alcohols, etc. as previous ly observed experimentally (Rahalkar et al., 2012). A capacity for utilizing various monosaccharides such as mannose, fucose, ribose, arabinose, rhamnose, xylose, and fructose was also observed. The extra cellular polysaccharides of benthic diatoms are commonly composed of rhamnose, fucose, xylose, mannose, galactose, glucose, and other mono mers (Bahulikar and Kroth, 2008). These sugar monomers are also found in natural biofilms dominated by diatoms (Bahulikar, 2006). The genome also exhibited genes for utilization of $\mathrm{N}$ acetyl glucos amine, chitin, myo inositol, putrescine, ethanolamine, glycerol, alginate which are amongst the most important common naturally occurring carbon substrates in the sticky biofilm environments (Sutherland, 2001). The chemotaxis genes and flagellar apparatus were detected which are particularly important in the biofilm environment for move ment towards $C$ sources.

Azospirillum is the next phylogenetic relative of Elstera after Lacibacterium, as revealed in NCBI blast. Genomes of Azospirillum species have been studied for their evolutionary origin (Wisniewski Dye et al., 2012) as they are the only terrestrial organisms amongst the Rhodospirillaceae family. Azospirillum is one of the most well stud ied organisms for its association with important cereals and grasses. In a detailed comparative genome analysis of Azospirillum spp. it was sug gested that it might have diverged much later than the suggested Pre cambrian split of 'hydrobacteria' to 'terrabacteria'. Also, the separation of Azospirillum from their close aquatic relatives coincided with the emergence of vascular plants on land (Wisniewski Dye et al., 2011). Bacteria associated with stone biofilms such as E. litoralis, can also be also explored as a possible link for the transition from aquatic to terres trial habitats, as they are present physically on the borderline connecting land and water. Thus, detailed study of $E$. litoralis genome can further shed light on the evolutionary divergence of 'hydrobacteria' and 'terrabacteria'. Azospirillum genome has acquired nearly half of its genes by horizontal transfer from terrestrial organisms like Burkholderia species. In preliminary analyses, we checked the origin for some of the genes in E. litoralis. We observed some of the genes showing ancestral origin, e.g. genes involved in the TCA cycle, nirB, flagella, DNA gyrase, etc. In contrast a few of the other genes, involved in the utilization of sugars, etc. showed similarity to Burkholderia, Rhizobium etc. indicating horizontal gene transfer (details not shown). A detailed study compar ing the Elstera genome with genomes of other members of the family Rhodospirillaceae including Azospirillum will help us to better under stand these interesting aspects of microbial evolution.

\section{Nucleotide sequence accession number}

The draft genome sequence of E. litoralis was deposited in the DDBJ/ EMBL/GenBank database under accession number LAJY00000000.1 (version 01).

\section{Acknowledgments}

This work was supported by Department of Biotechnology under the DBT BioCARe program (BT/BioCARe/06/840/2012) sanctioned to M. C. R. and fellowship sanctioned to P. S. P. is acknowledged. CSIR research fellowship to Preeti Arora is sincerely acknowledged.

\section{References}

Aziz, R.K., Bartels, D., Best, A.A., et al., 2008. The RAST server: rapid annotations using subsystems technology. BMC Genomics 9, 75.

Bahulikar, R., 2006. Diatoms From Littoral Zone of Lake Constance: Diversity, Phylogeny, Extracellular Polysaccharides and Bacterial Associations (Thesis). University of Konstanz, Konstanz.

Bahulikar, R., Kroth, P.G., 2008. The complex extracellular polysaccharides of mainly chain forming freshwater diatom species from epilithic biofilms. J. Phycol. 44, 1465-1475.

Baldani, J.I., Videira, S.S., dos Santos Teixeira, K.R., et al., 2014. The Family Rhodospirillaceae. pp. 533-618.

Bruckner, C.G., Bahulikar, R., Rahalkar, M., Schink, B., Kroth, P.G., 2008. Bacteria associated with benthic diatoms from Lake Constance: phylogeny and influences on diatom growth and secretion of extracellular polymeric substances. Appl. Environ. Microbiol. $74,7740-7749$.

Chevreux, B., Wetter, T., Suhai, S., (1999) Genome sequence assembly using trace signals and additional sequence information. vol. 99 (eds.), pp. 45-56. Hanover

Overbeek, R., Olson, R., Pusch, G.D., et al., 2014. The SEED and the Rapid Annotation of microbial genomes using Subsystems Technology (RAST). Nucleic Acids Res. 42 D206-D214

Rahalkar, M., Bahulikar, R.A., Deutzmann, J.S., Kroth, P.G., Schink, B., 2012. Elstera litoralis gen. nov., sp. nov., isolated from stone biofilms of Lake Constance, Germany. Int J. Syst. Evol. Microbiol. 62, 1750-1754

Sheu, S.Y., Chen, Y.L., Young, C.C., Chen, W.M., 2013. Lacibacterium aquatile gen. nov., sp. nov., a new member of the family Rhodospirillaceae isolated from a freshwater lake. Int. J. Syst. Evol. Microbiol. 63, 4797-4804.

Sutherland, I., 2001. Biofilm exopolysaccharides: a strong and sticky framework. Microbiology 147, 3-9.

Wisniewski-Dye, F., Borziak, K., Khalsa-Moyers, G., et al., 2011. Azospirillum genomes reveal transition of bacteria from aquatic to terrestrial environments. PLoS Genet. 7, $1-13$.

Wisniewski-Dye, F., Lozano, L., Acosta-Cruz, E., et al., 2012. Genome sequence of Azospirillum brasilense CBG497 and comparative analyses of Azospirillum core and accessory genomes provide insight into niche adaptation. Genes (Basel) 3, 576-602 